\title{
Erratum to: Western US high June 2015 temperatures and their relation to global warming and soil moisture
}

\author{
Sjoukje Y. Philip ${ }^{1} \cdot$ Sarah F. Kew $^{1} \cdot$ Mathias Hauser $^{2} \cdot$ Benoit P. Guillod $^{3}$. \\ Adriaan J. Teuling ${ }^{5}$ Kirien Whan ${ }^{1} \cdot$ Peter Uhe $^{3,4} \cdot$ Geert Jan van Oldenborgh ${ }^{1}$
}

Published online: 20 August 2017

(C) Springer-Verlag GmbH Germany 2017

\section{Erratum to: Clim Dyn \\ DOI 10.1007/s00382-017-3759-x}

In the original publication, the bar down the Fig. 7c-e has not been colored. The bar should be colored. The original article has been updated accordingly.

The online version of the original article can be found under doi:10.1007/s00382-017-3759-x.

Sjoukje Y. Philip

philip@knmi.nl

1 Royal Netherlands Meteorological Institute (KNMI),

De Bilt, The Netherlands

2 ETH Zürich, Zurich, Switzerland

3 Environmental Change Institute, University of Oxford, Oxford, UK

4 Oxford e-Research Centre, University of Oxford, Oxford, UK

5 Hydrology and Quantitative Water Management Group, Wageningen University, Wageningen, The Netherlands 
Fig. 7 Land atmospherecoupling metrics and anomaliesfor June 2015. a metric, bVAC index, c sensible heat fluxminus potential sensible heatflux anomaly $\mathrm{H}^{\sim}-\mathrm{H}^{\sim} \mathrm{p}$, d temperatureanomaly $\mathrm{T}^{\sim}$ and $\mathbf{e}$ latentheat flux anomaly $\mathrm{LH}^{\sim}$ a

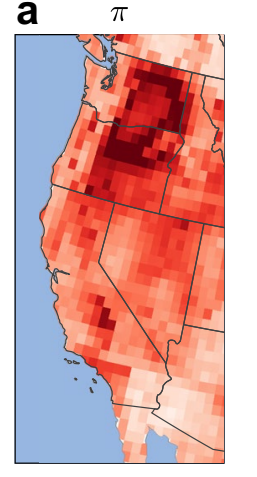

b VAC

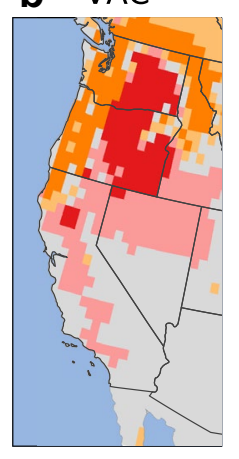

c $\mathrm{H}^{\prime}-\mathrm{Hp}^{\prime}$

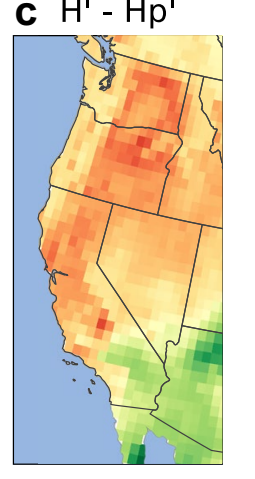

d $\mathrm{T}^{\prime}$

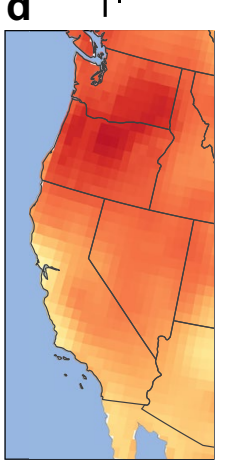

e $\mathrm{LH}^{\prime}$

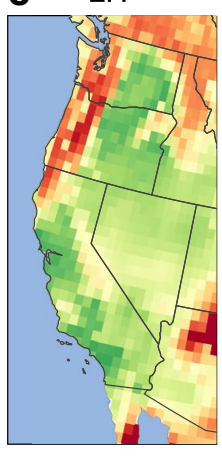

0.00 .51 .01 .52 .0

a $\quad b \quad c \quad d-$

$\begin{array}{llll}-2.0 & -1.5 & -1.0 & -0.5\end{array}$

$1.5 \quad 2.0$ 